Rev. Elev. Méd. Vét. Pays trop., 1967, 20, 4 (579-587)

\title{
Observation sur l'emploi du Prosalt d'antrycide et la résistance du bétail aux trypanosomiases
}

par R. COOLS

\begin{abstract}
RÉSUMÉ
Au Congo Kinshasa, dans un élevage à dominance Afrikander situé en région trypanosomée, le croît d'un troupeau a depuis cinq ans été systématiquement traité à titre préventif au Prosalt d'Anirycide, à l'àge de 9-12 et 18 mois.

La comparaison des résultats des examens de sang à l'état frais effectués sur l'ensemble des animaux de l'élevage semble indiquer que l'effet de ce produit dépasse sa simple action préventive.

En effet, les animaux du troupequ traité sont, lorsqu'âgés de 30 à 36 mois, significativement plus résistants aux trypanosomiases locales que ceux des troupeaux non traités.

Cette résistance accrue est attribuée à la prémunition chimı-biologique qui s'instaurerait au cours de la période où les jeunes animaux sont sous l'effet protecteur du Prosalt d'antrycide.
\end{abstract}

L'élevage où a eu lieu cette observation est situé au Katanga dans la région appelée «Lomani», à savanes plus ou moins boisées, avec galeries forestières hébergeant Gl. palpalis et $G$. fusca vectrices de $T$. vivax et surtout de T. congolense.

Il est divisé en six sections (consulter la carte jointe) comportant chacune des groupes de 120 d 160 animaux suivant la catégorie du bétail, qui vivent en vaine pâture de $6 \mathrm{~h}$ à $18 \mathrm{~h}$ ef sont parqués la nuit dans des « kraals 》 de 4 hectares environ.

Les trypanosomiases animales, qui entravent à la fois l'élevage et le développement économlque et social de ces régions, ont de tout temps constitué la préoccupation essentielle des éleveurs.

Contre les glossines, après abandon des zones par trop infestées, tous les moyens classiques habituels ont été utilisés (déboisement partiel ; pièges Harris; nébulisation d'insecticides au swing-fog, etc...) avec des résultats trop relatifs ou temporaires pour être vraiment efficaces.

Contre les trypanosomes, il a surtout été fait appel au traitement curatif (Bérénil ou Antrycide) des animaux reconnus atteints à l'occasion de mesures de dépistage automatiques par examen de sang à l'état frais, et à la chimio-prophylaxie grâce au Pro-salt d'Antrycide pour les animaux appelés à vivre en régions reconnues comme très infestées.

C'est ainsi que depuis 1962, dans la section de Mondole, la plus infestée de l'élevage, le P. A. est systématiquement utilisé chez le crôt et uniquement ainsi que suit :

- à 9 mois (au sevrage) : 1re injection à raison de $4 \mathrm{mg} / \mathrm{kg}$;

- à 12 mois : $2^{e}$ injection à raison de $4,4 \mathrm{mg} /$ $\mathrm{kg}$;

— à 18 mois : $3 \mathrm{e}$ injection à ralson de $4,4 \mathrm{mg} /$ kg.

par voie sous-cutanée au fanon, de façon à mettre les jeunes animaux à l'abri de la maladie pendant la période la plus critique de leur existence.

Cette méthode nous a donné les meilleurs résultats puisque l'état général de ces animaux 


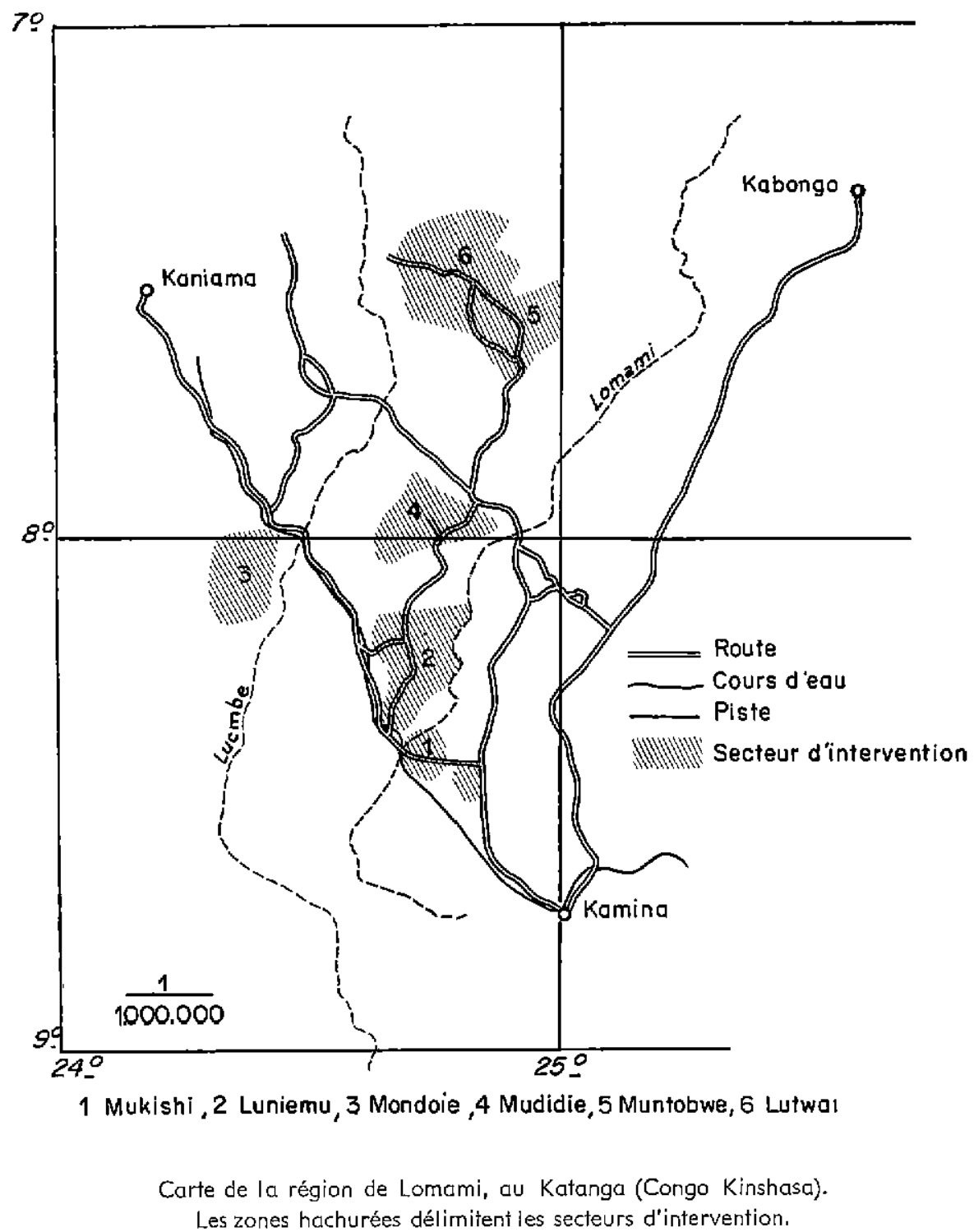


s'est amélioré avec une très sensible diminution de la mortalité générale passée de 5,3 p. 100 en moyenne entre 1959 et 1961, d̀ 1,7 p. 100 en moyenne entre 1962 et 1966 :

\begin{tabular}{|c|c|c|c|}
\hline 1959 & 3,8 p. 100 & $1963 \ldots$ & 1,05 \\
\hline & 5,4 p. 100 & $1964 \ldots$ & 1,3 \\
\hline 196 & 6,6 p. 100 & $1965 \ldots$ & 1,4 \\
\hline 1962 & 2,6 p. 100 & $1966 \ldots$ & 1,7 \\
\hline
\end{tabular}

En matière de dépistage des trypanosomiases la méthode de l'examen de sang à l'état frais n'a certes qu'une valeur relative mais dans le cas de nos observations elle prend une valeur certaine du moment qu'elle est appliquée uniformément, systématiquement et de façon analogue d̀ l'ensemble des animaux de l'élevage.

Le tableau i concrétise les résultats annuellement obtenus avec le pourcentage des cas positifs par rapport au nombre d'examens pratiqués ef par rapport au nombre moyen d'animaux que nous désignons comme «non réfractaire» c'est-à-dire la totalité du bétail moins les veàux réputés naturellement résistants qui n'ont pas fait l'objet d'examens de sang, ef moins les animaux soumis à l'activité préventive du Prosalt.

TABLEAU $N^{\circ} \mathrm{I}$

\begin{tabular}{|c|c|c|c|c|c|c|c|c|c|}
\hline Section & & 1959 & 1960 & 1961 & 1962 & 1963 & 1964 & 1965 & 1966 \\
\hline \multirow{4}{*}{ 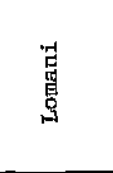 } & a & 41.878 & 41.862 & 24.448 & 24.665 & 24.375 & 26.770 & 33.308 & 43.356 \\
\hline & $b$ & 958 & 1.710 & 1.344 & 921 & 1.500 & 2.007 & 2.183 & 2.774 \\
\hline & c & 2,3 & 4,1 & 5,5 & 3,7 & 6,1 & 7,5 & 6,6 & 6,4 \\
\hline & d & 5,3 & 9,5 & 7,5 & 5,2 & 8,7 & 11,7 & 12,7 & 16,2 \\
\hline \multirow{4}{*}{ 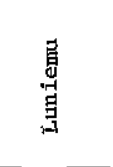 } & a & 4.622 & 4.586 & 2.473 & 2.504 & 2.415 & 2.682 & 1.970 & 4.556 \\
\hline & $\mathrm{b}$ & 209 & 117 & 215 & 57 & 178 & 120 & 183 & 467 \\
\hline & c & 4,5 & 2,5 & 8,7 & 2,3 & 7,4 & 4,5 & 9,3 & 10,2 \\
\hline & $d$ & 9,3 & 5,2 & 9,5 & 2,5 & 7,9 & 5,3 & 8,1 & 18,3 \\
\hline \multirow{4}{*}{ 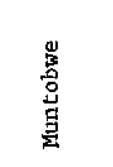 } & a & 9.401 & B. 652 & 1.618 & 3.433 & 5.509 & 7.064 & 10.456 & 13.168 \\
\hline & b & 170 & 224 & 138 & 297 & 506 & 735 & 890 & 997 \\
\hline & c & 1,8 & 2,6 & 8,5 & 8,6 & 9,2 & 10,4 & 8,5 & 7,6 \\
\hline & d & 3,7 & 4,8 & 2,9 & 6,4 & 10,9 & 15,8 & 19,1 & 21,4 \\
\hline \multirow{4}{*}{ 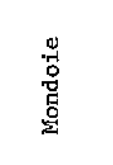 } & a & 9.045 & 11.201 & 9.713 & 7.299 & 4.864 & 6.792 & 5.838 & 4.750 \\
\hline & b & 212 & 740 & 450 & 210 & 97 & 87 & 48 & 77 \\
\hline & $c$ & 2,3 & 6,6 & 4,6 & 2,9 & 2,0 & 1,3 & 0,8 & 1,6 \\
\hline & $d$ & 8,9 & 31,1 & 18,9 & 10,0 & 6,3 & 5,7 & 3,2 & 5,0 \\
\hline
\end{tabular}

a) nombre d'examens pratiquês ; b) nombre de cas positifs ; c) pourcentage des examens positifs ;

d) pourcentage des cas positifs par rapport au nombre moyen d'animaux considérés comme "non rếracta1res"

Ces chiffres montrent, à partir de 1962, année où a débuté le traitement systématique préventif du croît du troupeau de Mondoie, au sevrage, une très nette amélioration tant par rapport à l'ensemble des animaux non traités que par rapport aux résultats antérieurement obtenus dans cette même section.

\section{INTERPRÉTATION DES RÉSULTATS}

La différence observée entre la période s'étendant de 1959 à 1961 et la suivante peut théoriquement s'expliquer soit par :

\section{a) Une diminution de la population des glossines.}

Cette hypothèse reste peu valable car sur le plan écologique, le milieu où ont évolué les animaux est resté identique à lui-même tout au long de la période d'observation. En outre, le Pro-salt est reconnu comme dépourvu de toute action insecticide vis-à-vis des glossines. Nousmêmes avons maintenu en vie pendant 25 jours 
plusieurs glossines nourries à 15 reprises sur des taureaux imprègnés de $P$. A. (voir détails de l'observation en annexe i) confirmant ainsi les résultats déjà publiés dans ce sens par ROUBAUD (E.) (16).

On pourrait penser d̀ une action antiglossinıenne des acaricides, utilisés dans les bains antitiques, SUTER $\left(H_{1}\right)(20)$ ayant démontré l'existence d'une telle action avec une rémanence de 2 jours pour les produits à base d'H. C. H. et de 10 jours pour ceux à base de D. D. T.

Utilisant depuis 1959, tant dans la section de Luniemu que dans celle de Mondoie, le Thiophosphate de chlorocoumarine (Asuntol Bayer) comme acaricide, nous nous sommes vainement employés d̀ mettre en évidence une possible action insecticide de ce produit vis-à-vis des glossines au cours d'expériences dont le détail figure en annexe I à cette note.

\section{b) Une diminution de l'infectivité des glossines.}

Cette hypothèse ne doit pas être à priori excive ne serait-ce que parce que ROUBAUD $\left(E_{1}\right)$ a obtenu une réduction de 60 p. 100 des infections salivaires chez des glossines nourries sur des animaux imprégnés de Pro-salt à raison de 5 et $10 \mathrm{mg} / \mathrm{kg}$ et bien que des expériences ultérieures ne lui aient pas permis d'obtenir un effet « désinfectant» aussi appréciable.

D'ailleurs dans les conditions où nous avons opéré on ne peut concevoir que des glossines s'infectent sur des animaux sous protection du Pro-salt du moment que sous son action les parasites disparaissent totalement de la circulation périphérique. Par contre, le pourcentage de glossines infectées doit dıminuer au fur et à mesure que du bétail sous protection remplace soit le petit gibier, soit le bétail non protégé, réservoirs possibles de parasites.

Ainsi les animaux traités préventivement ne doivent pas être considérés comme une réserve de contamination bien que cet état de prémunition chimique puisse aller de pair avec une certaine infection cryptique ainsi que cela a été mis en évidence par FIENNES (R.) (5).

\section{c) Une augmentation de la résistance du bétail.}

C'est là qu'd̀ notre avis réside la raison essentielle de la différence marquée observée sur le troupequ de Mondoie dès utilisation du Pro-salt comme trypanopréventif.

Tant pour vérifier ce fait que pour en préciser la nature et l'importance, nous nous sommes livrés à l'expérience suivante :

Le 15 septembre 1966, nous avons constitué

TABLEAU $\mathrm{N}^{\circ}$ II

Résultats et 11 mites de $I^{r}$ intervalle de conflance.

(Coëfficient de confiance, 95p.100)

\begin{tabular}{|c|c|c|c|c|c|c|c|c|}
\hline \multirow{2}{*}{ Gloupes } & \multicolumn{2}{|c|}{ Lot } & \multicolumn{2}{|c|}{ Lot } & \multicolumn{2}{|c|}{ Lot } & \multicolumn{2}{|c|}{ Totaux A } \\
\hline & Positufs & p.100 & Positifs & p. 100 & -Positifs & p. 100 & Positifs & p. 100 \\
\hline Luniernu & \multicolumn{2}{|c|}{ L1 } & \multicolumn{2}{|c|}{$\mathrm{L} 2$} & \multicolumn{2}{|c|}{$\mathrm{L} 3$} & \multicolumn{2}{|c|}{$\mathrm{L} 1+\mathrm{L} 2 \div \mathrm{L} 3$} \\
\hline Résultats & $10 / 20$ & 50 & $19 / 20$ & 95 & $17 / 20$ & 85 & $46 / 60$ & 77 \\
\hline Minimum & 5,4 & 27 & 17 & 85 & 12,4 & 62 & 39,6 & 66 \\
\hline Maximum & 14,6 & 73 & 20 & 100 & 19,4 & 97 & 51,8 & 86 \\
\hline Mondoie & \multicolumn{2}{|c|}{ M1 } & \multicolumn{2}{|c|}{$\mathrm{M2}$} & \multicolumn{2}{|c|}{$M 3$} & \multicolumn{2}{|c|}{$\mathrm{MI}+\mathrm{M} 2+\mathrm{M} 3$} \\
\hline Résultats & $2 / 20$ & 10 & $8 / 20$ & 40 & $10 / 20$ & 50 & $20 / 60$ & 33 \\
\hline Minimum & 0,25 & 1 & 3,8 & 19 & 5,4 & 27 & 13 & 22 \\
\hline Maximum & 6,3 & 32 & 12,8 & 64 & 14,6 & 73 & 28 & 47 \\
\hline Totaux B & \multicolumn{2}{|c|}{$\mathrm{M} 1+\mathrm{Ll}$} & \multicolumn{2}{|c|}{$\mathrm{M} 2+\mathrm{L} 2$} & \multicolumn{2}{|c|}{$\mathrm{M} 3+\mathrm{L} .3$} & \multicolumn{2}{|c|}{ Tota1 } \\
\hline Rẽsultats & $12 / 40$ & 30 & $27 / 40$ & 67,5 & $27 / 40$ & 67,5 & $66 / 120$ & 55 \\
\hline Minimum & 6,6 & 17 & 20,4 & 51 & 20,4 & 51 & & \\
\hline Maximutn & 18,6 & 46,5 & 32,6 & 81 & 32,6 & 81 & & \\
\hline
\end{tabular}


tant à Luniemu qu'à Mondoie un troupeau de 60 génisses de 30 à 36 mois chacun d'eux composé de lots de 20 génisses de Mondoie, de Luniemu et de Lutwai.

Nous disposions done :

à Mondoie (M) de 20 génisses de Mondoie (M1), 20 génisses de Luniemu ( $M 2$ ),

20 génisses de Lutwai (M3). à Luniemu $(L)$ de trois lots identiques .... L 1 aınsi désignés ........ L 2

qui toutes les deux semaines au moins ont été soumises à un examen de sang à l'état frais ; au 31 décembre chaque animal avait été examiné à 9 reprises.

Les résultats obtenus sont consignés dans le tableau II qui donne le nombre des animaux parasités par rapport au lot (20), ainsi que les limites de l'intervalle de confiance (coefficient 95 p. 100) (7).

Ils sont en outre donnés en pourcentage sous forme du graphique suivant, afin de faciliter les comparaisons.

Pour en faciliter la lecture il faut par exemple lire :

Luniemu - lot L 1 :

10 animaux trypanosomés sur 20 soit 50 p. 100.

- limite maximum suivant tableaux statistiques :

$14,6 / 20$ ou 73 p. 100 ,

- limite minimum suivant tableaux statistiques:

5,4/20 ou 27 p. 100.

A noter que deux génisses du lot $L 2$ et une du lof $L 3$ ont été trouvées infectées deux fois, une des deux du lot $L 2$ a même été trouvée infectée une troisième fois, ce dernier cas constituant l'unique infection à $T$. vivax constatée.

Chaque cas positif de frottis à l'état frais a fait l'objet d'un examen coloré, qui a toujours confirmé le premier à l'exception de deux cas du lot $M 1$.

\section{INTERPRÉTATION DES RÉSULTATS}

\section{I. - Comparaison en fonction du milieu : totaux A}

$$
\begin{aligned}
M 1+M 2+M 3 & : 33 \text { p. } 100 \\
& - \text { limites } 22 \text { ef } 47 \text { p. } 100
\end{aligned}
$$

$$
\begin{aligned}
\mathrm{L} 1+\mathrm{L} 2+\mathrm{L} 3 & : 77 \text { p. } 100 \\
& \text { - limites } 66 \text { et } 86 \text { p. } 100
\end{aligned}
$$

La différence entre le nombre des bêtes trypanosomées à Mondoie et celui observé à Luniemu est significative. Elle peut s'expliquer par la résistance du bétail, une fois mis à Mondoie ou ce qui paraît plus vraisemblable, pour une raison de milieu soit que les glossines y vivent moins nombreuses, soit qu'elles vivent moins infectées ou infectées avec une souche moins virulente, ces causes ayant par ailleurs pu jouer ensemble ou séparément.

Quoi qu'il en soit nous nous trouvons là devant une différence significative se situant dans le cadre de l'observation à laquelle les deux troupeaux ont été soumis, essal qui n'est par ailleurs pas nécessairement représentatif pour les deux sections considérées.

II. - Comparaison entre les origines des lots : totaux B

$$
\begin{aligned}
& \text { M1 L L } 1: 30 \text { p. } 100 \\
& \text { 一 limites } 17 \text { et } 46,5 \text { p. } 100 \\
& M 2+L 2: 67,5 \text { p. } 100 \\
& \text { - limites } 51 \text { et } 81 \text { p. } 100 \\
& \text { M3 + L } 3: 67,5 \text { p. } 100 \\
& \text { - limites } 51 \text { et } 81 \text { p. } 100
\end{aligned}
$$

Ces résultats montrent qu'il n'y a vraisemblablement pas de différence entre le bétail issu de Luniemu et celui issu de Lutwai.

Par contre la différence entre le bétail en provenance de Mondoie est significative.

\section{III. - Comparaison des origines par lot :}

Les différences entre $M 1$ et $L 1$ ainsi qu'entre L 3 ef $M 3$ ne sont pas significatives bien que les épreuves de $M$ et de $L$ soient différentes. Ceci semble indiquer que le simple transfert de bétail vers Mondoie n'a pas augmenté sa résistance.

\section{IV. - Comparaison par lot : par troupeau :}

Les comparaisons entre $M 1$ et $M 3$ et entre $L 1$ et $L 3$ indiquent que la valeur de la résistance est fonction de l'épreuve à laquelle ont été soumis les animaux.

Aucune spécificité éventuelle n'a pu être mise en évidence de façon significative.

Les comparaisons entre $M 2$ et $M 3$ et entre 
POURCENTAGE DE GÉNISSES TRYPANOSOMÉES ET LIMITES DE L'INTERVALLE DE CONFIANCE

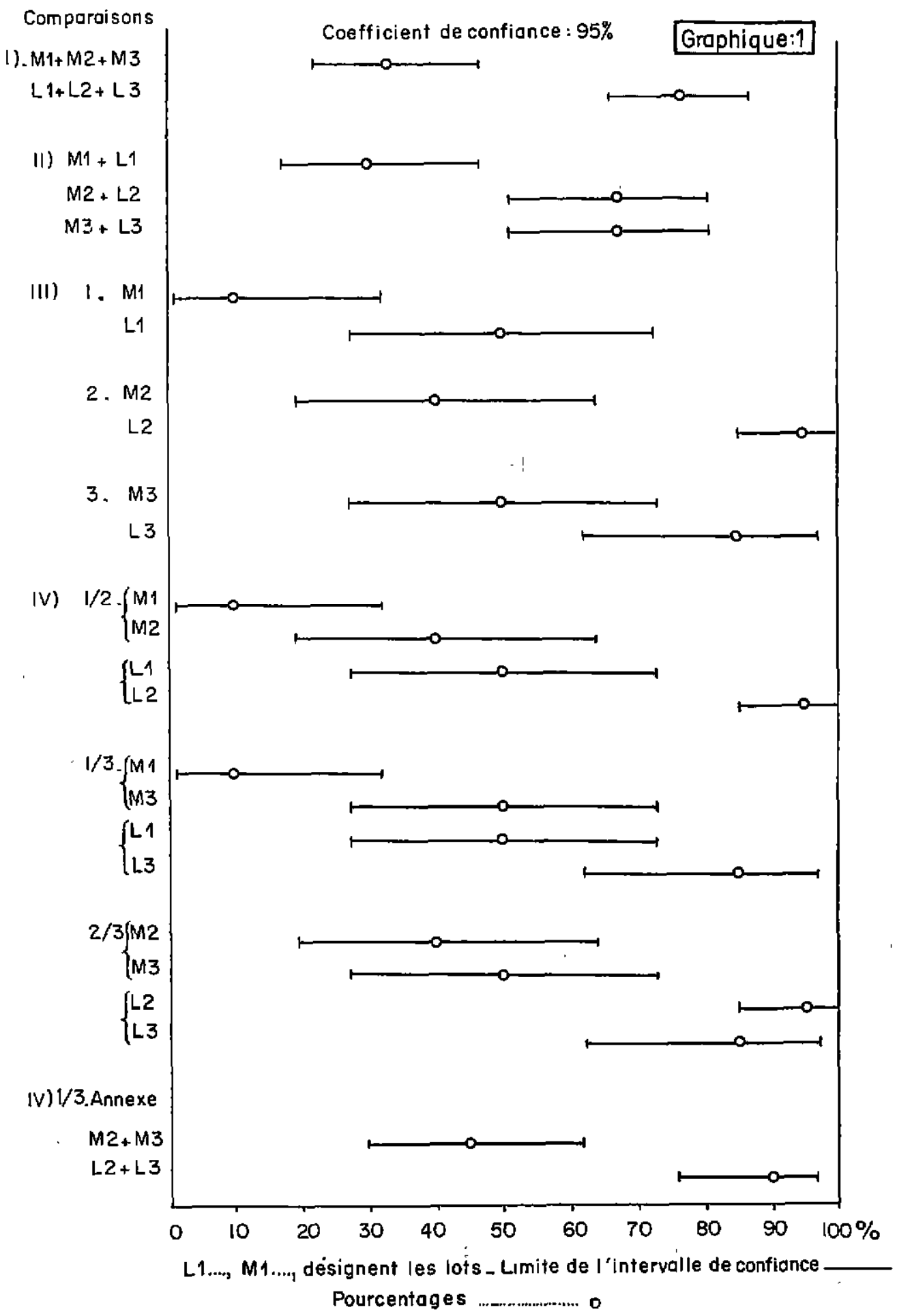


$L 2$ et $L 3$ montrent que le milieu d'origine n'a à lui seul pas provoqué de différence dans la résistance même pas à l'égard de la souche locale de trypanosome en admettant qu'elle existe en tant que telle.

\section{CONCLUSION}

A) Les observations faites ont mis en évidence l'existence d'une différence significative entre le taux d'infestation du bétail élevé à Mondoie et celui des animaux des autres sections.

Il est généralement admis que du bétail vivant depuis plusieurs générations en milieu trypanosomé acquiert un certain degré de résistance et plus précisément de prémunition contre la maladie. Cet état de prémunition pouvant être attribué à tout le bétail de l'élevage nous devons en conclure que c'est le Pro-salt qui, injecté à trois reprises au jeune bétail de Mondoie, a joué le rôle déterminant dans la résistance qu'il a manifesté de façon plus marquée contre la trypanosomiase.

Nous nous trouvons donc en présence d'un cas ou un produit chimique utilisé à titre préventif a permis au jeune bétail ainsi traité de développer une prémunition accrue que nous appelons avec d'autres auteurs prémunition chimio-biologique.

B) Une différence significative existe entre les épreuves de milieu auxquelles ont été soumis le troupeau d'expérience de Mondoie et celui de Luniemu. Cette différence peut s'expliquer par une possible différence d'infestivité des glossines. Mais ces essais et les résultats obtenus ne sont peut-être pas représentatifs pour les deux sections.

\section{ANNEXE I}

Action du Thiophosphate de chlorocoumarine sur la Glossina palpalis

\section{Introduction et résumé.}

Utilisant ce produit, sous sa forme commerciale (Asuntol Bayer), comme acaricide dans les dipping-tanks de l'élevage nous avons voulu contrôler sa possible influence parasiticide à l'égard des glossines se nourrissant sur le bétail baigné chaque semaine.
La conclusion est négative bien que l'Asuntol en émulsion à 0,05 p. 100 tue les glossines mises directement en contact avec elle.

\section{Expérience 1.}

- 24 glossines mises direciement en contact avec une émulsion de 0,05 p. 100 d'Asuntol meurent toutes dans les 4 heures qui suivent après avoir montré les signes d'intoxication classiques en la matière.

\section{Expérience II.}

- 54 glossines sont nourries sur un taureau $A$ traité au P. A. qui passe chaque semaine au bain d'Asuntol.

- 52 glossines sont nourries sur un taureau $B$ également au $P$. A. qui lui ne passe pas au bain.

- 5 glossines seulement nourries sur $A_{\text {, }}$ et 6 nourries sur $B$ meurent dans les 6 heures qui suivent leur mise en contact avec les animaux.

\section{Expérience III.}

Deux lots de glossines sont constitués en vue de les nourrir pendant plusieurs jours sur les deux taureaux.

- 5 glossines sont nourries tous les 2 jours sur le taureau $A$ et pareillement 6 sur le taureau $B$.

Après 10 jours d'observation deux glossines du lot $A$ ef trois du lot $B$ sont encore vivantes.

On ajoute alors respectivement 4 glossines neuves au lot $A$ et 3 au lot $B$.

15 jours plus tard après des repas tous les deux jours restèrent vivantes :

Pour le lot A 1 glossine du lot initial et 2 glossines sur les 4 ajoutées.

Pour le lot B 2 glossines du lot initial et 3 glossines sur les 3 ajoutées.

Il n'existe donc aucune différence sensible entre les deux lots de glossines, et une glossine est restée vivante pendant 25 jours bien qu'elle aif été 15 fois en contact avec le taureau $A$ passé au bain chaque semaine.

Cette expérience permet d'exclure toute action trypanocide de Pro-salt chez les glossines nourries sur du bétail traité avec ce produit.

\section{Conclusions.}

1) Le thiophosphate de chlorocoumarine 
(Asuntol Bayer) n'a d'action insecticide sur $G$. palpolis qu'à la condition qu'il y est contact direct avec le produit, en émulsion à 0,05 p. 100. La mortalité est alors de 100 p. 100 dans les quatre heures.

2) L'Asuntol n'a aucune action insecticide sur
G. palpalis si le contact a lieu par l'intermédiaire d'un animal baigné chaque semaine dans une émulsion à 0,05 p. 100.

L'Asuntol utilisé en bain acaricide ne peut donc être considéré comme constituant une arme valable dans la lutte contre les glossines.

\section{SUMMARY}

Observation on the use of Antrycide Prosalt and the resistance of cattle to Trypanosomiasis

In Congo Kinshasa, young cattle, mostly Afrikander from a herd living in an area infected with Trypanosomiasis, have been systematically treated with Antrycide Prosalt at the age of $9-12$ and 18 manths in order to prevent the disease.

The comparison of the results of the blood examinations carried out in these cattle showed that this drug had more than a preventive action and the animals which had been treated at the age of 30 to 36 months, were significantly more resistant to local Trypanosomiasis than those which had not been treated. This strengthened drug-resistance is supposed to be due to the chemio-biological premunition which would be set up during the period where the young animals are under the protection of Antrycide Prosalt,

\section{RESUMEN}

Observación sobre el empleo del Prosalt de antrycide y la resistencia del ganado a las tripanasomiasis

En el Congo Kinshasa, en una ganaderia principalmente constituida pro Afrikander y situada en una región donde se encuentra la tripanosomiasis, se está tratando sistemáticamente desde cinco años los animales de 9,12 y 18 meses de edad de modo preventivo con el Prosalt Antrycide.

La comparación de los resultados de los examenes de la sangre en el estado fresco en el conjunto de los animales de la ganaderia parece indicar que la acción de este producto sobrepasa su única acción preventiva.

En efecto, los animales de la ganaderia tratada, cuando tienen 30 a 36 meses de edad, son significativamente más resistentes a las tripanosomiasis locales que las de las ganaderias no tratadas. Se atribue esta resistencia a la premunición quimiobiologica que se instauraría durante el periodo cuando los jovenes animales estan bajo la acción protectora del Prosalt d'Antrycide.

\section{BIBLIOGRAPHIE}

1. CHANDLER (R. L.). - A study on cattle protected by Antrycide and exposed to regular challenges with trypanosomes. $J$. comp. Poth., 1958, $68: 261-63$.

2. CURASSON (G.). - Traité de protozoologie vétérinaire et comparée. Paris, Vigot, 1943.

3. DAVEY (D. G.). - Antrycide. A summary of informations, 1950.

- The chemotherapy of animal trypanosomiasis with particular reference to the trypanosomal diseases of domestic animals in Africa. Vet. Rev. Annot., 1957, $3: 15-36$.

4. DESOWITZ (R, S.). - Suramin complexes. II. Prophylactic activity against $T$. vivax in cattle. Ann. trop. Med. Parasit., 1957, 51 : 457-63.

- Studies on immunity and host-parasite relationships. I. The immunological response of resistant and susceptible breeds of cattle to trypanosomal challenge. Ann. trop. Med. Parásit., 1959, $53:$ 293-313. 
5. FIENNES (R. N. T.). - The cattle trypanosomiasis. Cryptic trypanosomiasis, 53.

6. HENNING (M. W.). - Animal diseases in South-Africa. Onderstepoort, Central news Agency, 1956.

7. LISON (L.). - Statistique appliquée à la biologie expérimentale. Paris, GauthierVillars, 1958.

8. MARQUES DA SILVA. - Expérience avec Antrycide à MAMBONE, 1952.

9. MORNET (P.), LALOINE (A.) et CISSIKA (M.). - Essai de trypano-prévention chimiothérapique des troupeaux de bovins d'exportation.

10. ORMEROD (W. E.). - A study of resistance to Antrycide in a strain of $T$. equiperdum. Brit. J. Pharmacol., 1952, 7 : 674-84.

11. PELLEGRINI (D.) et BONELLI (G.).Expérience sur la thérapie ef la chimioprophylaxie des trypanosomes par l'Antrycide, 1952.

12. PIRES (F. A.). - Aspects of the anti-glossinic fight in Mozambique. A control experiment of the $G$. brevipalpis Newst on the valley of the Maputo river. Portuguese East Africa. Bur. interafr. tsetse, B. P. I. T. T., $1950(143 / 0): 9$.

13. ROBSON (J.), WILDE (J. K. H.), - Prophylaxis against trypanosomiasis in zebu cattle, using Antrycide and DimidiumBromide, 1954.

14. ROBSON (J.). - A field trial of prophylactic drugs against trypanosomiasis in zebu cattle-Tanganyika. Vet. Rec., 1958, 70 : 925-27.

15. ROUBAUD (E.), BOURDIE (M.). - Essais sur l'action curative et préventive de l'Antrycide sur les trypanosomiases animales. Bull. Soc. Path. exot., 1950, 43 (9-10) : 552-56.
16. ROUBAUD (E.). - Recherches effectuées sur les glossines et infections trypanosomiennes dans les territoires africains de I'Union Française, 1950.

- Action de l'Antrycide sur les infections trypanosomiennes des tsé-tsés. Bull. Soc. Path. exof., 1952, 45 : 451-56.

17. SCHWETZ (J.). - Ełudes et notes d'entomologie médicale sur le Katanga, 27.

- Recherches sur les Glossines, 1919.

18. SMITH (I. M.). - The protection against trypanosomiasis conferred on cattle by repeated doses of Antrycide, alone or with T. congolense, 1958.

19. SOLTYS (M. A.), - Immunity in trypanosomiasis and its effect on chemotherapy. Vet. Rec., 1958, $70:$ 657-60.

- Sensivity of antibody-resistant strains to chemotherapeutic drugs. Parasifology, 1959. $49: 143-52$.

20. SUTER (H.). - Essai concernant l'application d'une émulsion de D. D. T. dans la lutte contre la tsé-tsé, 1947. Communications non-publiées.

21. THIENPONT (D.) et DEWOLF (H.).Contrôle en brousse de divers traitements de trypanosomiase chez les bovins, 1954.

22. UNSWORTH (K.). - Observation on Antrycide-fast-strain of $T$. congolense and $T$. vivax, 1955.

23. WEITZ (B. G. F.). - A soluble protective antigen of T. brucei. Nature, 1960, $185: 788$ 89.

24. WHITESIDE (E. F.). - The control of cattle trypanosomiasis with drugs in Kenya : methods and costs. E. Afr. agric. J., 1962, $28: 67-73$. 